\title{
2. Grazer Psychosentage - Erkrankungen aus dem schizophrenen Formenkreis - Schizophrenie und Affekt
}

Hans-Peter Kapfhammer

„Entscheidend ist, dass ... der Affekt ein relatives Übergewicht über die Seele erhält. Die Denkabläufe werden nicht mehr durch die Gesetze der Logik zusammengehalten, sondern durch die Logik der Affekte“.

Diese bereits 1911 von E. Bleuler hervorgehobene, vom Affekt dominierte Irrationalität im Erleben und Verhalten schizophrener Menschen fasziniert und erschreckt Laien wie Ärzte gleichermaßen. Der Blick auf die Schizophrenie als eine Störung des Affektes bzw. einer Störung der Integration von Emotionalität bestimmte das Motto der 2. Grazer Psychosentage (13. und 14. April 2007). In Analogie zur Gestaltung der 1. Tagung war intendiert, dieses Grundthema erneut aus ganz unterschiedlichen klinischen und theoretischen Perspektiven zu beleuchten und so zu einem besseren Verständnis des menschlichen und existenziellen Grunddilemmas beizutragen, das in dieser schweren seelischen Krankheit begründet liegt.

H. Fabisch und Kolleginnen führen in ihrem Referat grundlegend in die Affektthematik ein. Sie skizzieren drei unterschiedliche Untersuchungsmethoden, die psychopathologisch-phänomenologische, die verhaltenstheoretische und die psychoanalytische. Die phänomenologisch-psychopathologische $\mathrm{Zu}$ gangsweise spielt vor allem in den klassischen Texten der älteren Psychiatrie bei der Charakterisierung schizophrener Veränderungen eine vorrangige Rolle. Phänomenologische Ansätze finden aber auch in jüngeren Jahren wieder eine verstärkte wissenschaftliche Beachtung für eine Konzeptualisierung schizophrener Störungen. Durch die kognitive und emotionale Wende gelangt die Verhaltenstheorie zunehmend in den Anspruchsbereich von Theorie und Therapie und kann auch für die Behandlung von schizophrenen Patienten nutzbar gemacht werden. In der Psychoanalyse gelten die Affekte von Beginn an als Teil der psychischen Repräsentation von Triebenergie. In der weiteren psychoanalytischen Theorienentwicklung werden die Affekte als eine eigenständige Modalität des Unbewussten in ihrem Signal- und Kommunikationscharakter charakterisiert.

In seinem psychiatrie-historischen Beitrag kontrastiert $\boldsymbol{P}$. Hoff die jeweiligen theoreti- [2nd Graz Meeting on Psychoses - schizophrenia and affect] schen Grundpositionen E. Kraepelins und E. Beulers im Hinblick auf die pathogene Bedeutung der Affekte im schizophrenen Prozess. Wenngleich mit unterschiedlicher Akzentuierung waren sich Kraepelin und Bleuler einig, dass der affektive Bereich gerade bei den vermeintlich „nicht-affektiven“ Psychosen des schizophrenen Formenkreises ein zentrales Element darstellt. Hoff unterstreicht, dass in der aktuellen psychiatrischen Forschung die früher nosologisch orientierte Trennung von Kognition und Affekt weitgehend zugunsten eines syndrom- oder gar symptomorientierten Zugangs aufgegeben worden ist. Nach $\boldsymbol{F}$. Müller-Spahn steht der inhärente Zusammenhang von kognitiver und affektiver Störung auch im Zentrum der sog. „Paranoia-Diskussion“ seit den Anfängen der modernen Psychiatrie. Die grundlegenden Arbeiten der Tübinger Schule zum paranoischen Massenmörder Wagner durch Gaupp sowie zum sensitiven Beziehungswahn durch Kretschmer werden Modell bildend auch für spätere Beiträge. R. Gross belegt, wie fruchtbar psychoanalytische Affekttheorien für das Verständnis psychotischer, insbesondere auch schizophrener Störungen eingebracht werden können.

Das Thema „Affekt und Psychose“ lässt sich vortrefflich anhand zentraler Affektkonfigurationen diskutieren. C. Stuppäck und Koautorinnen illustrieren diese Vorgehensweise für die unterschiedlichen Gestalten der Angst bei Psychosen und H. G. Zapotoczky für die Dysphorie in ihrer Bedeutung für die psychotische Wut. W. Boeker schildert das existenzielle Dilemma vieler schizophren Erkrankter in der Erfahrung von Depression, Autonomieverlust und Suizid. H. P. Kapfhammer beschreibt grundlegende klinische und neurobiologische Aspekte von Postpartalpsychosen, die nosologisch einen engeren Konnex zu den bipolar affektiven als zu den schizophrenen Störungen erkennen lassen.

Lanzenberger und Kasper belegen, dass die modernen funktionellen und strukturellen Methoden des Neuroimaging einen weiterführenden neurobiologischen Einblick in den Zusammenhang von „Affekt und Psychose" verschaffen können. $N$. Müller demonstriert, dass psychoneuroimmunologische Befunde ebenfalls zu einer transnosologischen
Basis von schizophrenen und affektiven Psychosen beitragen. G. Klug betont eine transkulturelle Zugangsweise, die für das klinische Verständnis gerade von psychotischen Patienten mit Migrationshintergrund unverzichtbar ist. P. Hofmann und C. Ebner machen aufmerksam, dass in forensisch-psychiatrischen Begutachtungen von psychotischen Straftätern ein besonderes Augenmerk auf die zugrunde liegende Affektdynamik vor und während der Straftat zu richten ist.

W. Fleischhacker und A. Kaufmann geben einen Überblick über differenzielle psychopharmakologische Strategien bei schizophrenen Patienten, die zusätzlich eine affektive Komorbidität aufweisen. A. Baranyi und Kollegen widmen sich in ihrer Übersicht der Problematik des metabolischen Syndroms infolge einer psychopharmakologischen Behandlung und diskutieren notwendige Konsequenzen für das Compliance-Verhalten einerseits, für eine gesundheitsbewusste Lebensführung andererseits.

Abrundend spürt M. Musalek den möglichen Einflüssen von Melancholie, Manie und Psychose auf die Philosophiegeschichte nach. Und G. Langs beweist, dass die Psychopathologie des Wahnsinns in der Darstellung der Oper ohne den besonderen Rekurs auf große Emotionen blass und leer bliebe.

Ich möchte Frau Prof. K. Fabisch und Herrn Prof. H. Fabisch, aber auch Frau Prieler, Frau Schröttner und Herrn Mag. Fitz ganz herzlich danken. Sie haben mit ihrem großen Engagement die Planung und die Organisation der Tagung überhaupt erst ermöglicht.

Leider muss ich in diesem Vorwort auch an die traurige Realität erinnern, dass seit den 2. Grazer Psychosentagen zwei Referenten dieses Symposiums nicht mehr unter uns leben. Franz Müller-Spahn, Ordinarius und ärztlicher Direktor der Psychiatrischen Universitätsklinik Basel, ist am 4. August 2009 verstorben.

Und Hans Georg Zapotoczky, emeritierter Ordinarius und früherer Vorstand der Grazer Universitätsklinik für Psychiatrie ist am 3. Juli 2010 verstorben. Wir möchten beiden lieben Kollegen und Freunden auch mit diesem Symposiumsheft in großer Trauer gedenken. 